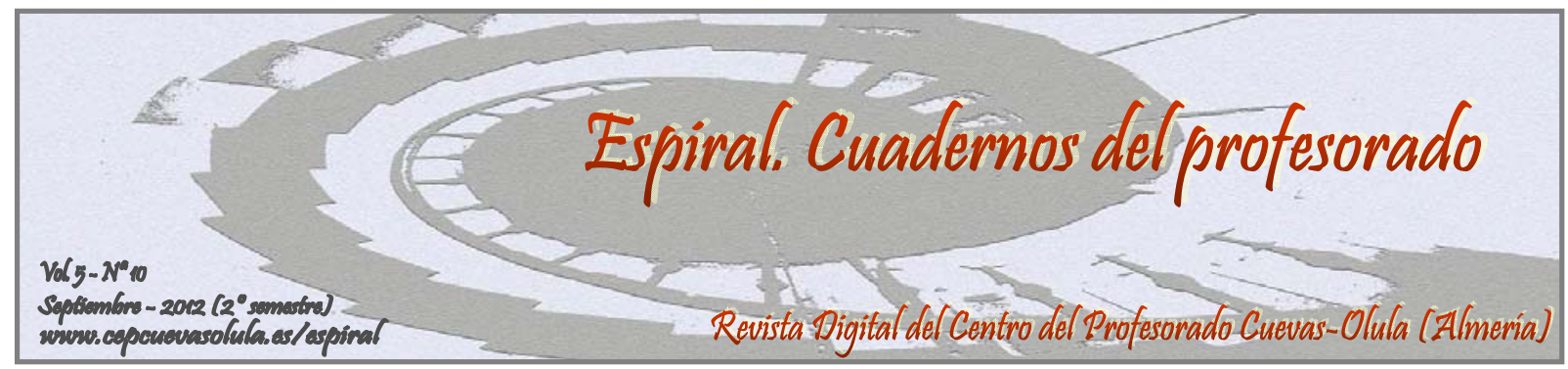

\title{
LA FORMACIÓN DEL PROFESORADO. UNA ASESORÍA COMPETENTE PARA UNA ESCUELA COMPETENTE
}

\author{
TEACHER TRAINING. COMPETENT ADVICE FOR A SCHOOL PROFICIENCY \\ Ana Alarcón Artero \\ Centro del Profesorado Cuevas-Olula, Junta de Andalucía, España
}

RESUMEN: El asesoramiento relacionado con los centros docentes ha ido evolucionando con el paso del tiempo desde de una concepción de asesoría experta y/o técnica, portadora de soluciones, hacia la adopción de un papel de acompañamiento basado en la búsqueda de soluciones conjuntas. En el modelo de asesoramiento va implícita la idea que se tiene de la educación y de la metodología a emplear, y esto afecta tanto a las asesorías de formación como al profesorado de los centros. Este posicionamiento necesita de un compromiso personal y una preparación que, podría pensarse, extralimita el dominio de los contenidos puramente didácticos. Estos razonamientos, incluyendo los cambios vertiginosos que se producen en el ámbito del conocimiento hacen más que necesaria la justificación de la formación permanente del profesorado. En este artículo se pretende aportar un modelo de intervención asesora surgido de las iniciativas del profesorado a partir de la revisión de las propias prácticas y de los resultados escolares para poder cambiar aquello que sea mejorable y de la necesidad de valorar su impacto e incidencia en los centros.

Palabras clave: asesoramiento, formación permanente, profesorado, intervención, práctica educativa.

ABSTRACT: The advice related to educational institutions has evolved over time from a conception of expert and / or technical advice which can give solutions towards adopting a role of support based on the searching for joint solutions. In the mentoring model is implicit the idea we have of education and methodology to be used, and this affects both consulting and training to teachers in the schools. This position requires a personal commitment and a preparation, one might think, oversteps the domain of purely didactic content. These arguments, including the rapid changes occurring in the field of knowledge do more necessary teacher training. This article aims to provide a model for intervention emerged advises faculty initiatives from reviewing their own practices and learning outcomes in order to change what is improved and the need to assess their impact and influence in the centers.

Key words: counseling, continuing education, teachers, intervention, educational practice.

Alarcón Artero, A. (2012). La formación del profesorado: una asesoría competente para una escuela competente. Espiral. Cuadernos del Profesorado, 5(10), 97-104. Disponible en: http://www.cepcuevasolula.es/espiral.

Fecha de recepción: 13/03/2012

Fecha de aceptación: 07/07/2012
Enviar correspondencia a: cepal3.ced@juntadeandalucia.es 


\section{1.- INTRODUCCIÓN. LA ASESORÍA Y LA FORMACIÓN PERMANENTE: REALIDAD Y DESEO}

La función asesora hoy en día nada tiene que ver con la del pasado, pero ¿en qué medida ha evolucionado su papel en la relación con el profesorado y los centros? Reemplazar ciertas ideas sobre el aprendizaje y sobre la educación en general, es más difícil de lo que se cree. Aceptar que no sólo se aprende dentro de un currículum establecido y que el aprendizaje tampoco se circunscribe al entorno escolar supone desequilibrar bastantes esquemas que se han ido aposentando a lo largo del tiempo. Los cambios tecnológicos y sociales suceden tan deprisa, que es necesario buscar nuevos enfoques, avanzando desde formas tradicionales a otras más eficaces.

Se hace necesario pasar de un modelo transmisivo y unificador a otro en el que se atienda a la diversidad, a la investigación, a la búsqueda y organización de la información, al desarrollo del pensamiento creativo y, en definitiva, a aprender a aprender. Esto supone un proceso de deconstrucción y construcción y tener en cuenta que intervienen aspectos académicos y no académicos. Los cambios vertiginosos que se suceden en los últimos tiempos y el enorme volumen de conocimientos existente nos lleva a pensar en la imposibilidad de retener siquiera una pequeña parte de ellos, por lo que ya no es tan necesario el almacenamiento en nuestras mentes sino saber buscarlo y hacer uso de él.

Quizá por estas razones, entre otras, podamos pensar que muchas de las profesiones de los niños y niñas que cursan primaria, aún no existan, entonces tendríamos que preguntarnos, tanto docentes como otros agentes educativos, qué papel jugamos en todo esto y si tenemos que seguir aferrados a metodologías incompatibles con estos principios, y en el mayor de los casos, evidencias; es decir, la escuela de antes no nos sirve ahora y mucho menos teniendo en cuenta la proyección de futuro, el alumnado y la sociedad de un mañana que está a la vuelta de la esquina y esto, os lleva a pensar en un compromiso ético por la mejora de la educación.

Entraríamos aquí en ese componente personal, en un posicionamiento sobre el tipo de educación, contenidos, métodos y estrategias adecuadas a la idea que cada cual tiene acerca del hecho educativo. Lo que resulta evidente es que estas nuevas corrientes derivadas de los cambios científicotecnológicos, culturales y/o políticos han ido conformando otros modelos y estilos de enseñanza, basados más en las necesidades del que aprende que en las del que enseña y no siempre son asumidas por el profesorado que se desorienta intentando aclimatarse a un cambio, al tiempo que está asomando otro y se abre más la brecha entre la preparación en su formación inicial a la que por cierto, habría que abordar de una vez por todas, y la realidad de las aulas y los centros, sobre todo ahora que hemos descubierto que dominar un temario, unos contenidos disciplinares teóricos con los que nos enfrentamos al hecho educativo, no nos lleva directamente a ser un o una profesional competente. El profesorado se ha visto forzado a adquirir, además, una serie de competencias tanto intrapersonales (autoconocimiento, autocontrol, autoestima, automotivación, estilo atribucional y resiliencia como interpersonales (asertividad, comunicación, empatía, gestión de conflictos, influencia o poder y negociación (Vaello, 2009). Según Goleman, (1966) dotado de inteligencia emocional. En una entrevista realizada a Bolívar alude a este tema argumentando que "en España se optó por no reformar la formación inicial del profesorado de educación secundaria y se ha mantenido durante cuatro décadas como si nada hubiese cambiado. Configurar una nueva etapa y olvidarse de la formación inicial ha supuesto un problema y un proceso de crisis de identidad para este profesorado. Parte de la labor de las asesorías se ha dirigido a suplir estas deficiencias” (Bolívar, 2012).

En este contexto, cobra su sentido la formación permanente del profesorado, tanto para adaptarse a los tiempos como para ir adquiriendo nuevas estrategias, ya que no se puede enseñar lo que no se tiene, no se sabe o no se comparte y, en muchos casos, perduran las formas de enseñanza recibida en el aprendizaje como alumno o alumna, más que lo aprendido en la formación docente (Arévalo, 2006). En todo caso, los docentes no siempre tenemos las soluciones para resolver los retos que se nos plantean.

Una de las instituciones relacionadas con la formación del profesorado más representativa son los Centros del Profesorado. Desde su creación en 1984 hasta hoy han ido pasando por una serie de 
cambios y dificultades. Tampoco han faltado las críticas señalándolos como meros ejecutores de los planes dictados por la administración educativa, sobre todo cuando en 1990 se explicitan las funciones y competencias del CEP dando paso a un proceso de burocratización de los mismos, convirtiéndose en meras herramientas de política educativa. Sin entrar en este debate, hemos de reconocer que el Centro del Profesorado, como institución de formación gestionada por el propio profesorado puede convertirse también en un potente medio formativo en la que el profesorado puede participar en su gestión y en la planificación, en la ejecución y evaluación de la formación permanente: no cabe duda de que esto supone un gran avance para los procesos de innovación desde la formación y en la formación y también por lo que se refiere al desarrollo profesional de todo el profesorado (Imbernón, 1994).

Cabe pensar que uno de los factores que interviene en esos procesos de cambio y mejora de la calidad educativa dentro de los Centros del Profesorado es el asesoramiento. Revisando la literatura al respecto vemos el papel que estos agentes externos han desempeñado a lo largo del tiempo, dependiendo este enfoque de la época y el autor, pasando desde una concepción tradicional en donde se piensa en una persona experta con grandes conocimientos que resuelve problemas hasta propuestas más actuales Bolívar (2005) en la que nos habla de que el asesoramiento debe plasmarse en un modelo de proceso de cambio como metodología de innovación y formación centrada en la escuela y otros autores (Masip, Teixidor y Vilalta, 2011) que proponen un asesoramiento de apoyo y compromiso con los centros y el profesorado, siendo el centro una organización que aprende. En términos similares se expresan otros autores como Hernández (2005) resaltando la labor de ayuda y apoyo, en el que están implicados múltiples agentes educativos y cuya finalidad es la mejora de la escuela a través de su capacitación y potenciación para la utilización de conocimiento pedagógico. Es decir, promover procesos que sean elaborados por el propio profesorado que va a participar en ellos. Podríamos reafirmar la idea de que se ha ido avanzando desde modelos aplicativos hacia modelos más implicativos. Es decir, la labor asesora debe ser de acompañamiento al docente, estableciendo vías de comunicación, espacios y tiempos donde se puedan poner en común las inquietudes y necesidades del profesorado. Igualmente, se hace necesario promover programas de tipo socio-emocional que permitan al profesorado adquirir las competencias emocionales, y que repercutirán en el cambio de todo el entorno de la comunidad educativa de cada centro.

De un tiempo a esta parte, se ha añadido al perfil anteriormente citado, poseer un dominio de las tecnologías de la información y la comunicación además de habilidades comunicativas y saber trabajar en equipo y colaborativamente, ya que la unidad de referencia es el Centro del Profesorado como institución y la asesoría también es miembro de un equipo.

Valgan estos ejemplos ya que no es el motivo de este trabajo realizar un paseo por la historia sino posicionarse en la idea que se tiene sobre el asesoramiento, pues de ella se derivarán las acciones que darán lugar a desarrollar uno u otro enfoque, teniendo esto una importancia crucial a la hora de la intervención en el centro educativo. Vemos así como el asesoramiento, como persona experta portadora de cambio ha dado paso a otros modelos centrados en el acompañamiento y compromiso con el centro en aras de una mejora de los rendimientos del alumnado y una mejor calidad de la educación. No debería acabarse este párrafo sin hacer alusión a la vertiente administrativa de las asesorías, en muchos casos mediatizada por su reglamentación en los distintos decretos y órdenes, independientemente de la comunidad autónoma que lo regule en el que se señala uno u otro perfil en función de las necesidades del Sistema. Sus funciones vienen prescritas normativamente.

\section{2.- HACIA UN MODELO DE ASESORAMIENTO}

El modelo de formación actúa siempre como currículum oculto de la enseñanza. La formación ha ido ligada a los conceptos que se tienen sobre la educación "Quien ejerce la educación se adscribe, implícita o explícitamente a una determinada concepción de esa práctica social y esto coincide con el concepto de sociedad y educación que se tiene” (Imbernon, 1994). 
En efecto, hemos visto que las escuelas necesitan cambiar para mejorar, tanto a nivel pedagógico como organizativo y que, aunque se consideren como unidades de cambio, la mayoría de las veces no pueden hacerlo solas. Sabemos también que los cambios requieren de nuevos planteamientos y prácticas pero que esto se consigue cuando el profesorado se implica en ello comprometiéndose profesional y personalmente. Los cambios sentidos desde fuera no suelen ser mirados con buenos ojos si no son propuestos internamente. También sabemos que estos cambios no ocurren de la noche a la mañana, desechando lo que ya existe ni a golpe de decretos, sino tomando conciencia de la necesidad de solucionar los obstáculos y las limitaciones que inciden en la práctica educativa sin caer en lo que denomina Alejandro Campo "ceguera colectiva": no ver que hay un problema, que el problema tiene solución y que la solución está en nuestras manos. (Campo, 2006) Las asesorías de formación, por su parte, deberían colaborar planteando estrategias y orientaciones que permitan al profesorado reflexionar y analizar la propia práctica dentro del contexto del centro encaminadas a la mejora; es decir, adoptar un papel de acompañamiento para que se desarrollen prácticas más autónomas mediante la reflexión de sus intervenciones y propuestas y a través de la reflexión de la propia práctica docente. Aprender a enseñar exige reflexión e investigación (Korthagen, 2011)

Si admitimos que hay una interrelación entre formación y desarrollo profesional y que concebimos el centro como una institución que aprende, entonces este será el lugar idóneo para desarrollar esa cultura profesional. La formación en los centros está considerada como la actuación formativa más eficaz y potente de cara a la introducción de cambios sustanciales en la enseñanza (del Carmen, 1995). El profesorado pasa de ser consumidor implementador y ejecutor de innovaciones de la formación, a diseñarla y gestionarla teniendo en cuenta su propia realidad desarrollando procesos de reflexión y una cultura colaborativa para contribuir a mejorar el proceso de enseñanza-aprendizaje y evaluar su impacto en la mejora de los rendimientos del alumnado.

Es evidente que no hay un recetario ideal sobre la intervención asesora. Será el contexto, la institución, el profesorado, quien determine la forma en que ésta se lleve a cabo y el motivo y circunstancias por las que el asesor o asesora entra en contacto con el centro ya sea a demanda o exigencia de la administración, querer solucionar o impulsar una temática concreta o perseguir un reconocimiento en forma de puntos.

La formación más efectiva es la que intenta dar respuesta a las demandas del profesorado a partir del propio contexto profesional, (Martín, 2005) huyendo de aquella en la que prima el reconocimiento asociado a la acumulación de méritos necesarios para cualquier cosa sin que necesariamente prime la innovación, el análisis critico, el desarrollo de competencias profesionales. Afortunadamente este tipo de actividades formativas han quedado obsoletas. La formación en centros se perfila como la modalidad más idónea para estos fines, porque está dirigida a equipos docente, se realiza con la intención de dar respuesta a las necesidades formativas detectadas por el profesorado de un centro para abordar una problemática concreta y contextualizada de la actividad docente. En este sentido, en los inicios de la LOGSE, se desarrolló en el País Vasco una línea de formación asentada en los propios centros y conocida como "Seminario de trabajo en Centros". Actualmente esta modalidad formativa sigue existiendo en diferentes comunidades autónomas con algunas diferencias de método entre ellas.

Una de las ventajas de la formación en centros es que permite evaluar el impacto de la formación, punto hacia donde se dirigen la mayoría de las críticas actuales, y hace imprescindible la realización del Plan de Formación del Profesorado si se quieren realizar demandas con sentido y saber lo que se ha avanzado según el punto de partida. Si no se revisan los resultados escolares, no se arbitran las estrategias que hay que poner en juego para mejorar, no se implica el centro, la comunidad y los diferentes agentes externos (inspección educativa, asesorías de formación y orientación), si esto no se hace así, estaremos perdiendo tiempo dando vueltas a una espiral sin salida y mirando hacia el lado equivocado. En el contexto de la formación permanente, la intervención de las asesorías resulta crucial. 


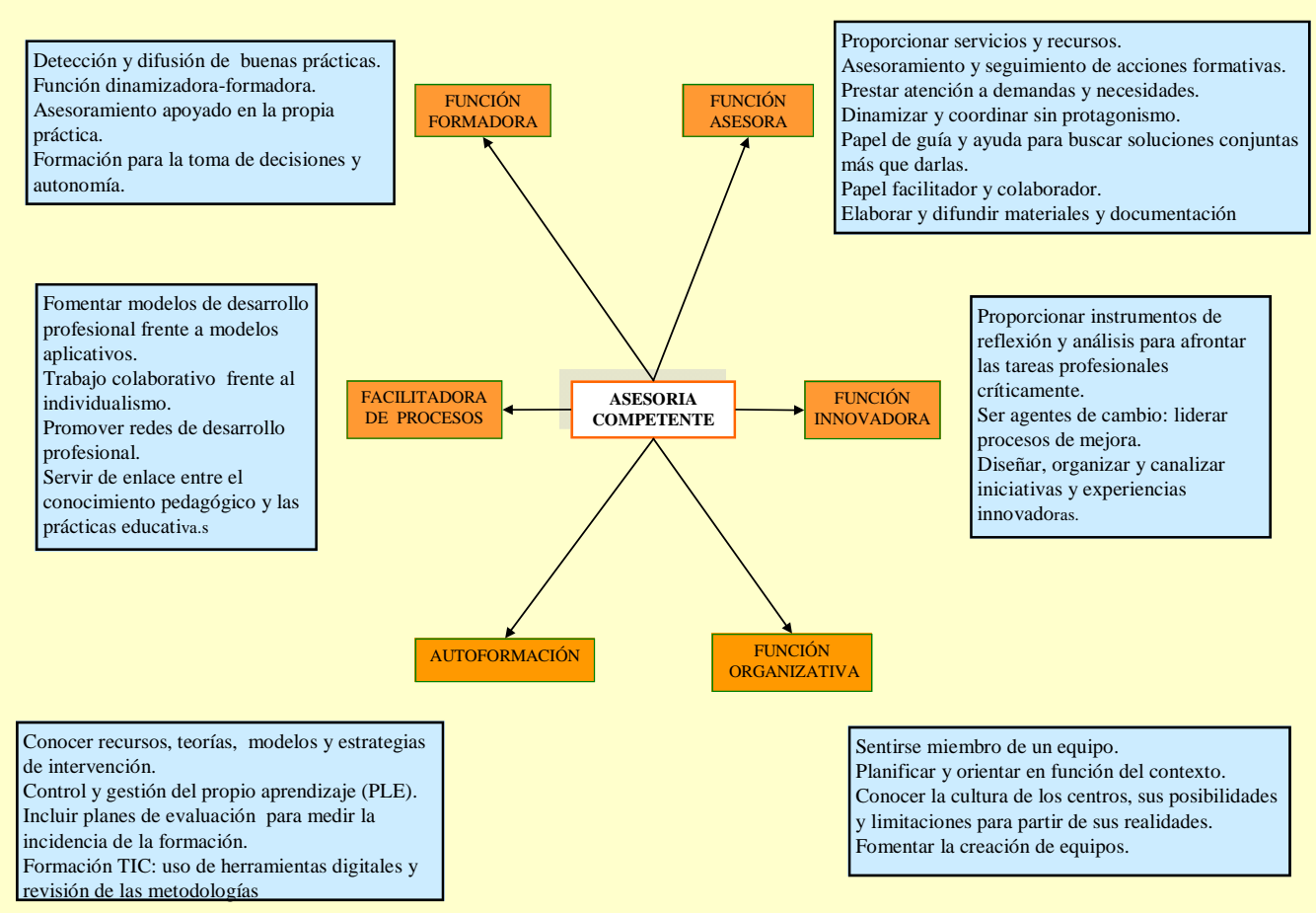

Figura 1.- Asesoría competente

Se propone una secuencia de actuación:

- Los centros docentes elaboran su Plan de Formación del Profesorado a partir de la revisión de los resultados escolares, autoevaluaciones y pruebas de diagnóstico, carencias e innovaciones para analizar y detectar las necesidades formativas con la voluntad de mejorar aspectos contemplados en el plan de centro.

- El equipo directivo, o quien determine la normativa, canaliza las demandas reales de formación relacionadas con el diagnóstico realizado en su centro hacia el Centro del Profesorado.

- Junto a la asesoría de referencia se concretan las demandas, se prioriza y delimita la temática y área de mejora, la modalidad formativa adecuada, los objetivos pretendidos, la temporalización y los recursos materiales y humanos necesarios (propios y externos) para llevarlo a cabo en función del profesorado implicado (lo ideal es que haya una implicación mayoritaria del centro, que se trabaje en un proyecto de formación en centros y se establezcan los horarios de trabajo en tiempo de obligada permanencia).

- La respuesta resultante debe ser negociada y consensuada entre las distintas partes delimitando responsabilidades, compromisos adquiridos y distribución de tareas.

- La asesoría debe entenderse como una ayuda planificada y realizarse de forma que en el plazo de tiempo más corto posible llegue a ser prescindible en relación a la tarea que se esté desarrollando (del Carmen ,1995).

- Una asesoría debe conocer las técnicas y estrategias necesarias que entraña el trabajo colaborativo y en equipo (ventajas e inconvenientes), la cultura del centro en el que interviene, los distintos intereses y niveles del profesorado y poseer las habilidades necesarias para reconducir al grupo sin imposiciones desde fuera.

- Análisis y valoración de los resultados, mecanismos de evaluación, el impacto y eficacia de la formación en el centro (incidencia en el aula y centro) en relación con los objetivos marcados y el área de mejora propuesta. La asesoría de responsable y el propio centro ela- 
boran los instrumentos necesarios para la evaluación del trabajo realizado teniendo en cuenta los logros conseguidos según el punto de partida para mejorar e implementar nuevas iniciativas.

\section{3.- CONCLUSIÓN}

Par ir concluyendo, vemos como el concepto de formación y asesoramiento ha sido el espejo en el que se ha ido reflejando el proceso de cambios producidos en la educación, no en vano han ido actuando como currículum oculto pasando de un marcado enfoque técnico-normativo-transmisivo a otro más actual en el que se piensa en una formación que se desarrolla dentro de una cultura profesional y en función de un contexto, desarrollo de competencias, procedimientos y estrategias necesarias para apoyar la intervención en las aulas. Según Vaello (2009), la necesidad de una triple preparación: psicológica, pedagógica y logística o la consideración del aprendizaje como un proceso continuo de construcción y reconstrucción consciente e inconsciente del entramado de representaciones emocionales (Pérez-Gómez, 2011) Estas reflexiones nos llevan a pensar en que un buen modelo de formación debe tener en cuenta el tipo de profesorado y prácticas educativas de cada centro las cuales van desde posturas meramente aplicativas e individualistas hasta otras más reflexivas, investigadoras y colaborativas con un profesorado diverso y centros entre sí diversos.

La experiencia docente nos enseña que la teoría por sí sola no representa un cambio automático en la práctica, ni siquiera la incorporación de las tecnologías de la información y la comunicación, tampoco la aparición de nuevos decretos, ni los modelos de formación traídos por agentes externos para que sean aplicados en el centro. La transformación debe producirse desde dentro, desde la reflexión, el compromiso y el deseo de cambio para la mejora.

Por último podría decirse que hablamos de eficacia de la formación cuando sabemos cómo afecta ésta al conocimiento, habilidades y actitudes del profesorado, cómo se mejora y transforma la cultura profesional y qué incidencia e impacto tiene todo esto en los resultados escolares y en la calidad de la educación en general. No podemos seguir realizando actividades formativas curso tras curso, repitiendo las mismas temáticas, para el profesorado de un centro cualquiera y no se tenga en cuenta su repercusión en las aulas. Esto merece una profunda reflexión por parte de toda la comunidad educativa.

\section{4.- REFERENCIAS BIBLIOGRÁFICAS}

Arévalo, T.E. (2006). Maestros para una escuela heterogénea. En Z. Jacobo. E. Adams y A. Ortiz, Sujeto, educación especial e integración. Vol. V, México: UNAM

Bolívar, A. (2005). Los procesos de asesoramiento: la experiencia de Ademe. Revista Electrónica Iberoamericana sobre Calidad, Eficacia y Cambio en Educación, 3, 1

Bolívar, A. (2012) El futuro de la formación del profesorado. E-CO, Revista digital de educación y formación del profesorado. E-CO, revista digital de educación y formación del profesorado de Córdoba, 9 (núm. extr.), 1-14.

Campo, A. (2006). El cambio en los centros educativos. Organización y gestión educativa 4, 1-8.

Carmen, L. del, (1992). La formación en centros y el asesoramiento. Material inédito.

Goleman, D. (1996). Inteligencia emocional. Barcelona: Cairos.

Hernández, V. (2005). Ayudando a nuestros centros a mejorar. Revista Electrónica Iberoamericana sobre Calidad, Eficacia y Cambio en Educación, 3, 1.

Imbernon F. (1994). La formación y el desarrollo profesional del profesorado. Hacia una nueva cultura profesional. Barcelona. Editorial Graó.

Korthagen, F.A. J. (2011). Principios para una formación eficaz. Cuadernos de pedagogía, nº 417, 56-59

Martín Rojo, E. (2005) La formación en centros: un modelo de formación permanente para equipos docente. Valencia: Nau Libres, edicions, culturals, valencianes.

Masip, M., Teixidor, M., y Vilalta, D. (2011) Acompañar procesos de cambio a través de la formación. Cuadernos de pedagogía, 417, 74-76. 
Pérez-Gómez, A.I. (2011) Aprender a educar(se): Una nueva ilustración para la escuela. Cuadernos de pedagogía, 417, 52-55.

Vaello, J. (2009) El profesorado socio-emocionalmente competente. Barcelona: Graó.

\section{NOTA}

Este artículo surge a raíz de la participación del centro del profesorado Cuevas-Olula en el programa ARCE (Agrupación de Centros Educativos) como centro asociado. El proyecto se denomina "la red de escuela competente" y está enmarcado dentro de la temática general "espacios de trabajo cooperativo a través de las TIC” 